\title{
Indicação e implementação de dispositivos de tecnologia assistiva a partir do uso do modelo teórico "Matching Person Technology (MPT)"
}

\section{Indication and implementation of technology devices assistive from the use of the theoretical model "Matching Person Technology (MPT)"}

\author{
Micaele Cunegundes de Souza Cândido ${ }^{1}$, Ana Cristina de Jesus Alves ${ }^{2}$
}

http://dx.doi.org/10.11606/issn.2238-6149.v29i2p155-163

Cândido MCS, Alves ACJ. Indicação e implementação de dispositivos de tecnologia assistiva a partir do uso do modelo teórico "Matching Person Technology (MPT)". Rev Ter Ocup Univ São Paulo. 2018 maio-ago.;29(2):155-63.

RESUMO: No Brasil, estudos evidenciaram lacunas nas fundamentações teóricas e nos instrumentos avaliativos específicos de tecnologia assistiva (TA). Objetivo: Indicar e implementar TA a partir do modelo Matching Person Technology e identificar os fatores que favorecem ou limitam este processo. Método: Trata-se de um estudo de quatro casos múltiplos descritivos de natureza qualitativa. Foi utilizado, a Avaliação da Predisposição ao Uso do Dispositivo de Tecnologia Assistiva e roteiro de observação. Os dados quantitativos foram analisados pela somatória dos escores, expressos em frequências e os qualitativos pela análise de conteúdo de Bardin. Resultados: Foram elaborados 1 caderno de comunicação alternativa (CA), 2 pranchas de CA, 1 pulseira de peso e 1 software para comunicação. Em $90 \%$ dos participantes a linguagem foi mencionada como limitação, em 100\% foi identificado fatores psicossociais positivos para o uso da TA e $90 \%$ tiveram altas expectativas em relação ao uso de TA. A observação mostrou fatores favoráveis (participação ativa nas escolhas, facilidade de uso, apoio da família) ou limitadores ao uso do dispositivo (frustração, dificuldade dos pais em aceitarem as escolhas e restrição social). Discussão e Conclusão: O modelo e a avaliação utilizados contribuíram para a escolha do dispositivo e no processo de implementação de TA.

Descritores: Equipamentos de auto-ajuda; Avaliação de processos e resultados (Cuidados de saúde); Reabilitação; Pessoas com deficiência; Terapia ocupacional.
Cândido MCS, Alves ACJ. Indication and implementation of technology devices assistive from the use of the theoretical model "Matching Person Technology (MPT)". Rev Ter Ocup Univ São Paulo. 2018 May-Aug.;29(2):155-63.

ABSTRACT: In Brazil, studies have revealed gaps in theoretical foundations and in the specific assessment instruments of the area of Assistive Technology (AT). Objective: Indicate and implement AT from the Matching Person Technology model and identify the factors that favored or limited this process. Method: It is a study of four cases with descriptive qualitative nature. We used the Assistive Technology Device Predisposition Assessment and observation script. The quantitative data were analyzed by the sum of the scores, expressed in frequencies and the qualitative ones by the Bardin content analysis. Results: 1 notebook of alternative communication (AC), 2 boards of $\mathrm{AC}, 1$ bracelet of weight and 1 software for communication were elaborated. In $90 \%$ of the participants, the language was mentioned as a limitation, $100 \%$ identified more positive factors for the use of AT and $90 \%$ had high expectations regarding the use of AT Observation showed favorable factors (active participation in the choices, ease of use, family support) or limiting the use of the device (frustration, parents' difficulty in accepting the choices and social restriction). Discussion and Conclusion: The model and the evaluation used contributed to the choice of the device and in the implementation process of AT.

Keywords: Self-help device; Outcome and process assessment (Health care); Rehabilitation; Disabled persons; Occupational therapy.

Este artigo é parte do Trabalho de Conclusão de Curso de Micaele C. S. Cândido apresentado à Universidade de Brasília - Faculdade de Ceilândia como requisito para obtenção do título de Bacharel em Terapia Ocupacional sob orientação da professora Dra. Ana Cristina de Jesus Alves.

1. Terapeuta Ocupacional, Universidade de Brasília. Residente do Programa Multiprofissional em Saúde Funcional e Reabilitação no Centro de Reabilitação e Readaptação Dr. Henrique Santillo. ORCID: https://orcid.org/0000-0002-5325-8772. Email: micaele_cunegundes@hotmail.com.

2. Docente do Programa de Pós-Graduação em Ciência da Reabilitação e do curso de Terapia Ocupacional da Universidade de Brasília - Faculdade de Ceilândia. ORCID: https://orcid.org/0000-0003-4361-1365. Email: crisjalves@hotmail.com.

Endereço para correspondência: QNN 14 Área Especial, 7380. Bairro Ceilândia Sul, Brasília, DF, Brasil. CEP: 72220140. E-mail: micaele_cunegundes@ hotmail.com. 


\section{INTRODUÇÃO}

$\mathrm{O}$ dispositivos de tecnologia assistiva (TA) são comumente indicados e implementados para pessoas com deficiências com intuito de favorecer a funcionalidade, autonomia, independência, qualidade de vida e participação social ${ }^{1}$.

Considerando as publicações nacionais sobre a temática da TA, estudos ${ }^{2}$ demonstraram que o foco das pesquisas orientam-se para a descrição de dispositivos de TA, o desenvolvimento de produtos e o desempenho do usuário com o dispositivo.

Por outro lado, a literatura científica internacional possui estudos para desenvolvimento de modelos teóricos, sistematização para implementação dos dispositivos de TA, investigação sobre fatores de abandono dos dispositivos implementados e no desenvolvimento de medidas avaliativas de eficácia ${ }^{3,4}$.

Ao considerarmos os instrumentos avaliativos para a indicação de dispositivos no cenário internacional, ganha destaque o uso de instrumentos específicos como a Quebec User Evaluation of Satisfaction with Assistive Technology (QUEST), Psychosocial Impact of Assistive Device Scale (PIADS), Individually Prioritised Problem Assessment (IPPA), Matching Person Technology (MPT), Assistive Technology Device Assessment (ATD-PA) e Matching Assistive Technology and Child (MACHT) ${ }^{4}$.

No Brasil, pesquisas ${ }^{4,5}$ evidenciaram lacunas quando comparados ao cenário internacional neste sentido. Um estudo de revisão ${ }^{5}$, que buscou por avaliações validadas no Brasil para prescrever TA, concluiu que nenhuma pesquisa utilizou avaliações específicas de TA. Os instrumentos aplicados por terapeutas ocupacionais, enfermeiros, educadores físicos e fisioterapeutas foram os seguintes: Escala de Berg, formulário semiestruturado criado por pesquisadores ou serviços, Questionário de Estado de Saúde (SF-36), Medida de Independência Funcional (MIF), Health Assessment Questionnaire (HAQ) e Furllentton Fitness Test (TAFI), tais, mensuram o uso do dispositivo em múltiplos aspectos.

No entanto, sabe-se que, recentemente, foi validada para uso no Brasil a avaliação de TA, a Quebec User Evaluation of Satisfaction with Assistive Technology (QUEST 2.0) ${ }^{6}$, que visa verificar a satisfação do usuário em relação ao dispositivo e ao serviço prestado em TA. Nesta mesma direção, Lourenço ${ }^{7}$ também propôs a validação nacional para o instrumento Physical Characteristics Assessment - Computer Access for Individuals with Cerebral Palsy (PCA), que permite caracterizar os métodos de acesso e a entrada de informações no computador a partir das habilidades motoras das pessoas com paralisia cerebral. Com o objetivo de buscar evidências científicas no Brasil para indicação e implementação de dispositivos de TA, identificou-se um estudo ${ }^{8}$ que propôs um fluxograma para o desenvolvimento de dispositivos ${ }^{8}$ e pesquisas com abordagens práticas que descreveram apontamentos para nortear o uso de TA e favorecer o desempenho da pessoa com deficiência no contexto escolar ${ }^{9,10}$. Foi encontrado, também, o modelo teórico Matching Person and Technology $(\mathrm{MPT})^{3}$, validado recentemente no Brasil, mas nunca utilizado como embasamento teórico em estudos nacionais ${ }^{3}$.

"O modelo MPT consiste em uma série de medidas desenvolvidas para dirigir e organizar as várias influências que impactam o uso da tecnologia na assistência, educação, trabalho e cuidados na saúde, incluindo fatores pessoais e sociais" (p.8) .

O modelo Matching Person and Technology tem abordagem individualizada e centrada na pessoa. Sua proposta é auxiliar o profissional e o usuário de TA a identificarem, conjuntamente, necessidades, preferências, motivação do usuário e seu estilo de vida para o melhor uso da TA. De acordo com o modelo, 3 fatores devem nortear a indicação e o uso da TA: os fatores psicossociais, ambientais e do equipamento são considerados fundamentais para uma boa combinação entre a pessoa e a tecnologia ${ }^{3,11}$.

Os fatores psicossociais do indivíduo referem-se às características como: motivação, cooperação, otimismo, paciência, autodisciplina, experiências positivas de vida, habilidades para o uso e a percepção entre a situação desejada e a atual motivação. Os fatores ligados ao ambiente associam-se ao apoio familiar, dos pares, do empregador, e aos ajustes que recompensem o uso do dispositivo e a exigência externa. Já as características do dispositivo de TA dizem respeito à habilidade de ser utilizada sem desconforto ou estresse, sua compatibilidade com outras tecnologias, seu custo, a credibilidade do dispositivo, sua transportabilidade e facilidade para uso no presente e futuro ${ }^{3}$.

Com isso, o objetivo deste estudo foi indicar e implementar dispositivos de tecnologia assistiva a partir do referencial teórico específico da área, o Matching Person and Technology (MPT). Pretendeu-se, também, identificar os fatores que favoreceram ou limitaram este processo.

\section{PROCEDIMENTOS METODOLÓGICOS}

Trata-se de um estudo de casos múltiplos descritivos de natureza qualitativa. Neste método são abordados vários casos para a investigação de determinado fenômeno com ampla descrição em seu contexto ${ }^{12}$.

Neste estudo, foi elencado a amostra de conveniência ${ }^{12}$ 
onde os casos são selecionados com base em critérios previamente estabelecidos. Com isso, os participantes submetidos a pesquisa deveriam ter disponibilidade para as seções semanais, interesse em utilizar dispositivos de TA para auxílio em suas atividades de vida diária, comunicação aumentativa e alternativa, dispositivos de acessibilidade ao computador e adequação postural.
Participaram desta pesquisa quatro sujeitos com deficiência física que foram encaminhados pela equipe interdisciplinar da Associação de Centro de Treinamento de Educação Física Especial (CETEFE) e/ou pelo Núcleo de Tecnologia Assistiva, Acessibilidade e Inovação (NTAAI).

O Quadro 1 apresenta a caracterização dos participantes.

Quadro 1 - Caracterização dos participantes

\begin{tabular}{|c|c|c|c|c|c|}
\hline $\mathbf{P}$ & Sexo & Idade & Diagnóstico & Comunicação & Atividades de vida diária (dependente) \\
\hline 1 & Fem. & 5 & $\begin{array}{l}\text { Paralisia cerebral } \\
\text { tetraparesia espástica }\end{array}$ & Linguagem não verbal & $\begin{array}{l}\text { Vestir } \\
\text { Alimentação } \\
\text { Mobilidade Funcional } \\
\text { Banho } \\
\text { Uso do vaso sanitário e realização de higiene intima e } \\
\text { pessoal } \\
\text { Cuidado com equipamentos pessoais }\end{array}$ \\
\hline 2 & Fem. & 5 & $\begin{array}{l}\text { Paralisia cerebral } \\
\text { tetraparesia espástica }\end{array}$ & Linguagem não verbal & $\begin{array}{l}\text { Vestir } \\
\text { Alimentação } \\
\text { Mobilidade Funcional } \\
\text { Banho } \\
\text { Uso do vaso sanitário e realização de higiene íntima e } \\
\text { pessoal } \\
\text { Cuidado com equipamentos pessoais }\end{array}$ \\
\hline 3 & Masc. & 6 & $\begin{array}{l}\text { Artrogripose múltipla } \\
\text { congênita }\end{array}$ & Linguagem verbal & $\begin{array}{l}\text { Vestir } \\
\text { Alimentação } \\
\text { Mobilidade Funcional } \\
\text { Banho } \\
\text { Uso do vaso sanitário e realização de higiene íntima e } \\
\text { pessoal } \\
\text { Cuidado com equipamentos pessoais }\end{array}$ \\
\hline 4 & Masc. & 28 & $\begin{array}{l}\text { Paralisia Cerebral } \\
\text { diparesia espástica }\end{array}$ & Linguagem não verbal & $\begin{array}{l}\text { Banho } \\
\text { Uso do vaso sanitário e realização de higiene íntima e } \\
\text { pessoal } \\
\text { Cuidado com equipamentos pessoais }\end{array}$ \\
\hline
\end{tabular}

Nota: ${ }^{1} \mathrm{P}=$ participante

Observa-se homogeneidade dos sexos e a faixa etária com variação de 5 a 28 anos. Dentre os quatro participantes, três apresentavam diagnóstico de paralisia cerebral e possuíam linguagem não verbal. Referente as atividades de vida diária, todos os participantes eram dependentes em alguma área.

A coleta dos dados ocorreu no CETEFE, no período de setembro a novembro de 2016, com dez seções semanais realizadas por cinco pesquisadores, que receberam treinamento sobre o MPT e para aplicação dos instrumentos utilizados nesta pesquisa, no Grupo de Estudos de Terapia Ocupacional e Tecnologia Assistiva (ToTec) da Universidade de Brasília.

Como preconizado pela literatura científica ${ }^{12}$, para 
a coleta de dados foram aplicados múltiplos instrumentos com os responsáveis e/ou com o participante, contanto que a expressão e a compreensão estivessem coerentes.

Primeiramente, foi utilizado o formulário de caracterização do participante criado pela pesquisadora, a fim de obter informações sobre sexo, idade, diagnóstico, comunicação utilizada e, também, foram investigadas as áreas de desempenho do usuário em atividades de vida diária como, vestir-se, alimentar-se, mobilidade funcional, banho, uso do vaso sanitário, higiene íntima e pessoal e cuidados com os equipamentos pessoais.

Posteriormente, foi aplicada a Avaliação de Tecnologia Assistiva-Predisposição ao Uso (ATD PA Br) ${ }^{3}$ que compõe o modelo MPT. Tanto a avaliação quanto o MPT foram traduzidos e validados para uso com livre acesso ${ }^{3}$ no Brasil, e têm o objetivo de investigar os potenciais dos participantes para o uso bem-sucedido de TA e possibilitar a combinação ideal entre o usuário e tecnologia. Foram utilizados o formulário do Cliente e do Dispositivo.

A partir do formulário do Cliente ${ }^{3}$ foram avaliados três domínios. O primeiro buscou investigar as habilidades funcionais dos participantes ( 9 itens) por meio de uma escala de 1 (ruim) à 5 (excelente) e a necessidade de TA para cada habilidade. O segundo identificou a satisfação em áreas de desempenho e a participação (12 itens) com pontuação de 1 (não satisfeito) a 5 (muito satisfeito). $\mathrm{O}$ terceiro considerou os fatores psicossociais dos participantes (33 itens) como barreiras (-) ou facilitadores $(+)$ ao uso.

O formulário do Dispositivo ${ }^{3}$ quantificou as expectativas em relação ao uso da tecnologia assistiva apontada (12 itens). Seu escore é obtido pela soma dos itens indicados por meio da escala de 1 (o tempo todo / $100 \%$ do tempo) a 0 (não se aplica) $)^{3}$.

Utilizou-se, também, a observação participante, em que o pesquisador se integra ao grupo visando prioritariamente a observação ${ }^{12}$. Assim, para o registro, após cada seção, foi preenchido o roteiro de observação qualitativo, desenvolvido pela pesquisadora, com o intuito de descrever as observações durante a seção, considerandose os itens norteadores descritos pelo MPT como: os fatores observados pelos pesquisadores ou trazidos pelos participantes, os aspectos relacionados ao dispositivo de TA utilizada, o contexto das seções ou do indivíduo e os resultados alcançados.

Os dados da ATD PA BR foram analisados por meio da análise quantitativa descritiva somando-se os escores e a frequência. No formulário do cliente, em habilidades funcionais, satisfação com desempenho e participação foram destacados os itens com menores escores (1), classificando, assim, as demandas para a intervenção. Nos fatores psicossociais, foram destacados a quantidade de itens considerados facilitadores (+) e as barreiras (-) ao uso da TA. Já no formulário do Dispositivo, os subitens correspondentes às expectativas em relação ao uso da TA foram somados, conforme indicado no manual ${ }^{3}$.

Os dados do roteiro de observação foram analisados através da análise de conteúdo de Bardin ${ }^{13}$, compreendendo as etapas de pré-análise, de exploração do material, de tratamento dos resultados, de inferência e de interpretação.

Este estudo faz parte da pesquisa intitulada Identificação e Proposição de uma Metodologia de Implementação de Recursos de Tecnologia Assistiva aprovada pelo Comitê de Ética e Pesquisa (CEP) da Universidade Federal de São Carlos (UFSCar) sob parecer número 045/2012. Todos os participantes e/ou responsáveis assinaram o termo de consentimento livre esclarecido.

\section{RESULTADOS}

O Quadro 2 apresenta os dispositivos de TA indicados e implementados a partir das demandas dos participantes e/ou de seu cuidador.

Quadro 2- Dispositivos indicados e implementados

\begin{tabular}{|cl|}
\hline Participantes & Dispositivo de TA \\
\hline 1 & Caderno de CA \\
2 & Prancha de CA de papel \\
3 & Pulseira com peso \\
4 & $\begin{array}{l}\text { Software de comunicação para tablete } \\
\text { Prancha de CA temática escolar }\end{array}$ \\
\hline
\end{tabular}

Observa-se que foram selecionados 5 dispositivos de TA: 1 caderno de comunicação alternativa (CA), 1 prancha de CA temática escolar, 1 pulseira de punho para auxílio na escrita e na alimentação, 1 software de comunicação para tablete e 1 prancha de CA de papel.

O Quadro 3 apresenta os escores mais baixos ligados às habilidades, satisfação com desempenho e participação, e aos fatores psicossociais dos participantes obtidos por meio da ATD PA Br. 
Quadro 3 - Habilidades, satisfação com desempenho e participação, e fatores psicossociais com menores escores dos participantes

\begin{tabular}{|c|c|c|c|c|}
\hline \multirow{2}{*}{ ATDA PA Br (escalas) } & \multicolumn{4}{|c|}{ Participantes } \\
\hline & 1 & 2 & 3 & 2 \\
\hline Habilidades Funcionais & $\begin{array}{l}\text { Linguagem (1) } \\
\text { Físico (1) } \\
\text { Uso da parte inferior (1) }\end{array}$ & $\begin{array}{l}\text { Linguagem (1) } \\
\text { Uso da parte } \\
\text { inferior (1) } \\
\text { Mobilidade (1) }\end{array}$ & $\begin{array}{l}\text { Preensão e uso } \\
\text { dos dedos (1) }\end{array}$ & Linguagem (1) \\
\hline $\begin{array}{l}\text { Satisfação com } \\
\text { desempenho e } \\
\text { participação em } \\
\text { atividades }\end{array}$ & $\begin{array}{l}\text { Saúde em Geral (1) } \\
\text { Autonomia e } \\
\text { autodeterminação (1) }\end{array}$ & $\begin{array}{l}\text { Cuidado pessoal } \\
\text { e atividades } \\
\text { domésticas (1) } \\
\text { Participação } \\
\text { em atividades } \\
\text { desejadas (1) }\end{array}$ & $\begin{array}{l}\text { Não foram } \\
\text { demarcados itens } \\
\text { de escore } 1\end{array}$ & $\begin{array}{l}\text { Não foram demarcados } \\
\text { itens de escore } 1\end{array}$ \\
\hline \multirow{2}{*}{ Fatores Psicossociais } & $17(+)$ & $\mathbf{X}$ & $13(+)$ & $19(+)$ \\
\hline & $2(-)$ & $\mathbf{X}$ & $1(-)$ & $1(-)$ \\
\hline $\begin{array}{l}\text { Expectativa em relação ao } \\
\text { uso do dispositivo }\end{array}$ & $\begin{array}{l}\text { CA (49) } \\
\text { Cadeira para escola (48) } \\
\text { Órtese (44) }\end{array}$ & $\mathrm{CA}(52)$ & $\begin{array}{l}\text { Adaptação } \\
\text { para escrita e } \\
\text { alimentação (44) }\end{array}$ & CA (41) \\
\hline
\end{tabular}

Nota ${ }^{1} \mathbf{X}=$ participante não respondeu, ${ }^{2} \mathrm{CA}=$ comunicação alternativa

Pode-se visualizar que, nas habilidades funcionais, 90\% dos participantes mencionaram a linguagem como limitação. Quanto à satisfação com desempenho e participação, 50\% dos participantes não demarcaram itens com pontuação ruim (1). Em relação às características psicossociais, todos os participantes tiveram pontuações superiores nos aspectos facilitadores para o uso da TA. Nas expectativas para uso do dispositivo, em 90\% dos participantes, a pontuação para o uso de comunicação alternativa foi superior as outras TA mencionadas.

A análise de conteúdo do roteiro de observação, preenchido pelos pesquisadores (PE) nas seções com os participantes (PA), resultou em três categorias: 1) Fatores pessoais do participante que podem influenciar o uso da TA; 2) Fatores do contexto e o uso de TA; 3) O dispositivo de TA, que serão descritas a seguir.

\section{CATEGORIA 1- Fatores pessoais do participante e o uso da TA}

Todos os participantes desta pesquisa demonstraram ter habilidades para o uso dos dispositivos indicados e implementados.

\footnotetext{
"A participante (PA2) respondeu positivamente em relação à escolha dos símbolos e figuras através do olhar" (PE2).

"Participante (PA 1) conseguiu utilizar os símbolos com facilidade, inclusive, realizando expressões faciais enquanto olhava para o símbolo" (PE1).
}

Durante as seções foram identificadas a motivação e a cooperação dos participantes.

\begin{abstract}
"Na sala de seção, a participante (PA1) mostrava-se motivada, participativa e interessada na utilização dos símbolos por meio da atividade proposta” (PE 1).

"Participante (PA4) trouxe uma lista de palavras e de frases para adicionar a prancha temática, demonstrando motivação para utilizar o dispositivo" (PE 2).
\end{abstract}

Alguns participantes demonstraram sono excessivo, desinteresse, resistência e facilidade de frustração. Tais características podem ser observadas nos relatos a seguir:

"Participante (PA2) demonstra desinteresse e resistência ao que lhe é proposto na seção mantendo a cabeça baixa" (PE2).

“Em alguns momentos, o participante (PA3) não conseguiu usar a caneta na pulseira. Então, pedia para usar a boca para apoiar a caneta. Quando negamos, ele pedia para fazer outra coisa, por exemplo, brincar com algum brinquedo e falava que estava cansado, se negando a continuar a atividade" (PE3).

"Nesta seção, a participante (PA2) estava com sono, o que influenciou sua participação efetiva na seção" (PE2).

Outro fator apresentado por todos os participantes foi a possibilidade de autonomia oferecida por meio do dispositivo nas fases iniciais do processo de implementação.

"Com isso, a participante (PA1) escolheu a história da Branca de Neve e mencionou, por meio dos símbolos, não 
querer contar a história para a mãe” (PE1).

"Mãe se mostrou frustrada com a participante (PA 2) quando esta relatou, por meio dos símbolos, não querer se comunicar pelo caderno com a mãe" (PE 2).

Observou-se a indecisão dos responsáveis ao traçar os seus objetivos prioritários.

“... previamente ao início das seções, a mãe havia elencado várias outras demandas para a participante (PA1), que não apareceram na aplicação dos questionários. A genitora, ainda, mostrou-se confusa sobre o objetivo das seções" (PE1).

\section{CATEGORIA 2- Fatores do contexto e o uso de TA.}

Identificou-se a dificuldade dos responsáveis em aceitar as escolhas dos filhos.

"Nesta seção, foi solicitado que a mãe aguardasse a filha em outro espaço para que a participante (PA2) fosse estimulada a se comunicar com outras pessoas e demonstrasse seu real desejo e potencial de comunicação. No entanto, a responsável demonstrou resistência a sair, mas, ao final da seção, mencionou surpresa pela filha ter ficado toda a seção sem sua participação” (PE2).

"No momento da escolha da pasta, a responsável, por vezes, não permitiu que o participante (PA4) escolhesse a estética de sua pasta temática. Neste sentido, ressaltase que o participante manifestou receio em contrapor a opinião da mãe, preferindo em grande parte, interiorizar suas preferências e priorizar as opiniões da mãe" (PE2).

O envolvimento e o interesse da família, a observação dos resultados alcançados através do dispositivo, a orientação sobre seu uso e o envolvimento na construção do dispositivo, ocasionam o aumento de sua credibilidade e motiva o seu uso.

"Quando a participante (PA2) olhou fixamente para o que queria naquele momento, a mãe se mostrou realizada pela evolução da mesma. Assim, no fim da seção, a mãe se mostrou solicita às orientações sobre a organização da prancha de comunicação e sobre a utilização da prancha de papel em casa" (PE2).

No entanto, constatou-se desinteresse de algumas famílias em observar os resultados alcançados por meio do dispositivo.

"A participante (PA1) mostrava-se motivada, participativa e interessada na atividade, porém, quando saiu da sala, mudou sua fisionomia na presença da mãe" ... "No fim da seção, a mãe não demonstrou muito interesse em conseguir entender o que a criança tentava relatar por meio da prancha" (PE1).
Além do ambiente familiar, outros locais onde o participante estava inserido permeavam o uso da TA.

"Mãe relata pouca interação do pai com a participante (PA1) devido à comunicação e, menciona preocupação quanto à filha na escola, pois os professores possuem dificuldades para entender o que a criança está comunicando" (PE1).

"Participante (PA2) mostrou-se pronta para introdução da prancha, mas a mesma não tem com quem utilizar o dispositivo, visto que ela não está indo para a escola e não demonstra interesse em comunicar-se com a mãe" (PE2).

\section{CATEGORIA 3- O dispositivo de TA.}

Os participantes descreveram característica do dispositivo que permitiam seu uso sem desconforto, estresse e que possibilitava o uso futuramente.

"Participante (PA3) conseguiu pintar, mas, para fazer o tracejado, foi necessário girar o caderno algumas vezes para ele conseguir alcançar o desenho e pintar. Com isso, observou-se que seria necessário utilizar um suporte móvel para que ele pudesse se movimentar e alcançar diversas partes de uma mesma folha" (PE3).

"Nesta seção, o participante (PA4) foi instruído e treinado para fazer adição de novas palavras, frases e categorias ao aplicativo de comunicação alternativa” (PE2).

Outro fator descrito, que permeia as características dos dispositivos, foi a possibilidade de transportar a TA em qualquer local desejado pelo participante.

"Durante a seção, o participante (PA4) relatou não estar utilizando a prancha na escola por receio de lhe roubarem o tablete. Com isso, foi confeccionada prancha temática escolar de papel" (PE2).

Alguns componentes do dispositivo de TA foram relatados como barreira ao uso.

"Percebeu-se que a quantidade de subpastas existentes no aplicativo compromete a agilidade da comunicação do participante (PA4). Então, foi realizado o desmembramento das pastas e foram adicionadas novas palavras" (PE2).

Em relação a elaboração da TA, houve a participação dos usuários na escolha do dispositivo, na seleção da estética e confecção durante a implementação.

"Os símbolos utilizados na seção foram colados no caderno junto com a participante (PA1)” (PE1).

"Nesta seção foram apresentados os tipos de comunicação alternativa e os aplicativos de CA disponíveis os quais poderiam corresponder às 
demandas do participante (PA4). Após isso, ele realizou a escolha de sua preferência" "Participante trouxe uma lista de palavras e frases por ação volitiva para adicionar em prancha temática, demonstrando engajamento no objetivo proposto" (PE2)..

\section{DISCUSSÃO}

Neste estudo, utilizou-se os nortes teóricos e a avaliação do modelo MPT, a ATD PA Br. Este instrumento tem sido referenciado como eficaz para verificar a predisposição para utilização de dispositivos de $\mathrm{TA}^{11}$. Da mesma forma, essa pesquisa mostrou que, o uso do instrumento permitiu aos participantes identificar suas habilidades, suas demandas, sua satisfação no desempenho nas atividades cotidianas e o uso da TA neste contexto.

Os resultados obtidos mostraram que os participantes com paralisia cerebral indicaram escore 1 (ruim) no item linguagem. Houve, também, maior escore na escala de expectativa para o uso do dispositivo de comunicação alternativa, demonstrando que, indivíduos com distúrbios severos na comunicação oral e escrita mostraram expectativas superiores em relação aos dispositivos de auxílio para comunicação ${ }^{14}$.

Em relação a escala de satisfação com desempenho e participação onde foram elencados os itens de insatisfação (1), diferentes itens para mudanças prioritárias foram identificados por $50 \%$ dos participantes, e em $50 \%$ dos participantes não foram pontuados itens de insatisfação. Estes resultados permitem inferir que, sujeitos com a mesma deficiência apresentaram satisfação no seu desempenho e em sua participação de forma diferenciadas, ocasionando demandas subjetivas, individuais e distintas no que diz respeito a funcionalidade desejada com o uso do dispositivo. Isto reforça a necessidade de avaliações e de observações rigorosas que preconizem a demanda centrada no indivíduo e em seu contexto na indicação e implementação do dispositivo ${ }^{3}$.

Quanto aos fatores psicossociais, em todos os participantes, as pontuações foram superiores nos pontos positivos relacionados ao uso da TA, tais como, ter visão de capacidades, qualidade de vida e bem-estar subjetivo, autodeterminação, humor e autoestima, motivação para o uso de TA e confiança no programa terapêutico ${ }^{3}$. Os resultados sugerem que os participantes possuíam fatores favoráveis (de predisposição) ao uso eficaz da TA. Scherer et al. ${ }^{11}$ mostraram que os fatores pessoais e psicossociais variam em cada sujeito e influenciam o uso da TA, assim como, estes fatores podem minimizar o abandono do dispositivo. Em acordo com estes resultados, autores ${ }^{3},{ }^{15}$ apontaram que a utilização eficaz do dispositivo ocorrerá quando houver cooperação, motivação, otimismo e habilidade de uso por parte do usuário.

Os resultados da análise qualitativa evidenciaram, assim como o modelo MPT e os estudos já realizados ${ }^{3,11}$, que os fatores pessoais do participante, tais como as habilidades necessárias para o uso do dispositivo, motivação, cooperação, sono excessivo, desinteresse, resistência, facilidade de frustração, possibilidade de autonomia e indecisão em relação aos objetivos prioritários, ocasionaram influências positivas e negativas durante o processo de indicação e implementação do dispositivo de TA.

Referente a influência dos fatores do contexto onde o participante estava inserido destacaram-se a dificuldade dos pais em aceitar as escolhas dos filhos, o envolvimento e/ou desinteresse da família, o aprimoramento das relações sociais e a utilização eficaz do dispositivo em ambientes desejáveis.

Os fatores do dispositivo de TA que influenciaram o processo foram: a utilização do dispositivo sem desconforto ou estresse; as características do dispositivo que possibilitam seu transporte e uso futuro; a participação ativa do usuário na escolha do dispositivo, seleção da estética e confecção.

Assim, os resultados reforçaram a necessidade da avaliação individual que analise os componentes do indivíduo em relação aos critérios: físicos, motores, cognitivos, sensoriais, emocionais e do desempenho ocupacional ${ }^{3,14}$.

Na análise qualitativa foram observadas características psicossociais negativas, tais como, desinteresse, resistência, facilidade de frustração e sono excessivo, diferentemente dos dados quantitativos. Este dado pode estar relacionado aos achados de Rocha et al. ${ }^{16}$, que evidenciaram a organização da rotina das famílias de crianças com paralisia cerebral. Essa rotina, muitas vezes, é estruturada de acordo com as terapias, que podem acontecer até quatro vezes por semana. A partir destas discussões, é possível pensar que as características psicossociais negativas descritas podem ter sido ocasionadas pelo cansaço dos participantes.

Com relação à dificuldade dos pais em considerar as escolhas de seus filhos e possibilitar a autonomia e a independência através de seu real potencial, autores ${ }^{19}$ evidenciaram que a superproteção dos pais de pessoas com deficiências favorece para que a autonomia e a independência não sejam alcançadas. Pesquisadores ${ }^{20}$ descreveram que pais superprotetivos tendem à subestimar as habilidades reais dos filhos.

Observou-se que a influência do posicionamento dos familiares dos usuários, em relação ao processo de implementação da TA, pode influenciar positivamente ou 
negativamente o benefício trazido pelo dispositivo. Em consonância com estes resultados, Alves ${ }^{21}$ destacou como fundamental o envolvimento da família e do usuário no processo de indicação e implementação para utilização eficaz do dispositivo, visto que a ausência deste apoio e a falta de treinamento dos familiares podem ocasionar o abandono do dispositivo, já que os cuidadores influenciam, negativamente ou positivamente, na utilização do dispositivo ao longo do tempo ${ }^{22}$.

Identificou-se, também, que a ausência das relações sociais dos usuários desmotivou o uso da TA. Estudos destacaram que a implementação dos dispositivos de TA pode trazer benefícios sociais ${ }^{14}$. Nesta direção, Pape et al. ${ }^{23}$ salienta que a integração eficaz entre tecnologia e usuários está vinculada aos benefícios sociais oferecidos.

Quanto a utilização eficaz do dispositivo em locais cotidianamente frequentados como a escola, estudos ${ }^{21}$ destacam que o uso contínuo do dispositivo auxilia na escolarização e na inclusão de pessoas com deficiências.

Em relação ao uso dos dispositivos, os roteiros de observação salientaram que a utilização do dispositivo sem desconforto e estresse influenciam o processo de implementação. Costa et al. ${ }^{15}$ descreveram fatores os específicos da TA que ocasionam o abandono, tais como, o peso elevado, a falta de funcionalidade, o excesso de ruídos, o desconforto e a dificuldade de uso, sendo que, para o item desconforto favorecerá a dor e insatisfação do usuário. Outra característica do dispositivo, que influencia seu bom uso, é sua disponibilidade de utilização em diferentes momentos e etapas da vida. Isso pode nos remeter que, dispositivos que não possuem características favoráveis para utilização futura poderão ser abandonados por inadequação ${ }^{3}$. Verza et al. ${ }^{24}$ demonstraram em seu estudo que $24,2 \%(n=54)$ eram abandonados por inadequação do dispositivo.

Referente, ainda, às características do dispositivo, foram verificadas influências quanto as particularidades da TA para seu transporte a locais desejáveis. Autores ${ }^{18}$ mostraram que pessoas com deficiência física/motora abandonavam o uso de dispositivos de difícil manuseio ou transporte.

Este estudo observou que a participação ativa do usuário na escolha do dispositivo, na seleção da estética e confecção são fatores de influência positiva. Porém, Hohmann e Cassapian ${ }^{25}$ verificaram que os dispositivos de TA estão sendo implementados sem levar em consideração a participação ativa do usuário. Por conseguinte, estudos mencionam que a ausência da participação ativa do usuário durante o processo descrito ocasiona a insatisfação e consequentemente o abandono do dispositivo ${ }^{15,11}$. A partir destes dados, discute-se que a participação do usuário durante o processo de indicação e implementação possibilita que o usuário perceba o dispositivo implementado como parte dele, possibilitando empoderamento para suas escolhas em relação ao uso de sua TA.

\section{CONCLUSÕES}

Os resultados desta pesquisa apontaram que o processo de indicação e implementação de dispositivo de TA necessita de abordagens individualizadas e centradas no sujeito, uma vez que, perpassa por aspectos do usuário, do contexto e da própria TA utilizada que influenciarão positivamente ou negativamente o uso e a satisfação do usuário.

Os apontamentos deste estudo podem contribuir apresentando o uso de um método, que ofereceu nortes teóricos e instrumentais, para indicar e implementar a TA, identificando seus fatores favoráveis ou suas barreiras ao uso.

Esta pesquisa possibilitou um aprofundamento nas discussões por meio do estudo de casos múltiplos sobre o tema dentro de uma abordagem específica. Sugere-se mais estudos na área, utilizando abordagens transversais com amostras maiores, além de pesquisas longitudinais, para compreendermos melhor como tem se dado a avaliação e a implementação de TA.

Agradecimentos: Agradecemos a Universidade de Brasília - Faculdade de Ceilândia (UNB FCE), ao Núcleo de Tecnologia Assistiva, Acessibilidade e Inovação do Distrito Federal (NTAAI) e a Associação de Centro de Treinamento de Educação Física Especial (CETEFE).

Autoria e Indicação de Responsabilidade: Micaele Cunegundes e Ana Cristina de Jesus Alves participaram da concepção, delineamento da pesquisa, escrita da pesquisa e análise dos dados. Micaele Cunegundes participou da coleta e sistematização dos dados. Todos os pesquisadores participaram da redação, da revisão do artigo e da aprovação da versão a ser publicada.

\section{REFERÊNCIAS}

1. Brasil. Secretaria dos Direitos Humanos. Subsecretaria Nacional de Promoção dos Direitos da Pessoa com Deficiência. Comitê de Ajudas Técnicas. Tecnologia Assistiva. Brasília: CORDE; 2009. Disponível em: http://www.pessoacomdeficiencia. gov.br/app/sites/default/files/publicacoes/livro-tecnologia- assistiva.pdf.

2. Braccialli LMP. Tecnologia assistiva e produção do conhecimento no Brasil. J Res Spec Educ Need. 2016;16(1):1014-7. doi: 10.1111/1471-3802.12355. 
3. Alves ACJ. Avaliação de tecnologia assistiva predisposição ao uso: ATD PA BR: versão brasileira. Brasília: Editora Universidade de Brasília; 2017. Disponível em: http:// repositorio.unb.br/bitstream/10482/31065/1/RELATORIO_ AvaliacaodetecnologiaassistivaATDPA.pdf.

4. Alves ACJ, Matsukura TS. Revisão sobre avaliações para indicação de dispositivos de tecnologia assistiva. Rev Ter Ocup Univ São Paulo. 2014;25(2):199-207. doi: 10.11606/ issn.2238-6149.v25i2p199-207.

5. Marques MPM. Avaliações para indicação de tecnologia assistiva utilizadas por terapeutas ocupacionais [monografia]. Brasília, DF: Universidade de Brasília; 2013. Disponível em: http://bdm.unb.br/bitstream/10483/7666/1/2013 MariannePinheiroMarques.pdf.

6. Carvalho KEC, Júnior MBG, Sá KN. Tradução e validação do Quebec User Evaluation of Satisfaction with Assistive Technology (QUEST 2.0) para o idioma português do Brasil. Rev Bras Reumatol. 2014;54(4):260-67. doi: 10.1016/j. rbr.2014.04.003.

7. Lourenço GF. Protocolo para avaliar a acessibilidade ao computador para alunos com paralisia cerebral [dissertação]. São Carlos: Universidade Federal de São Carlos; 2008. Disponível em: https://repositorio.ufscar.br/handle/ ufscar/2987?show=full.

8. Maia NM, Freitas SF. Proposta de um fluxograma para o processo de desenvolvimento de produtos de tecnologia assistiva. Cad Ter Ocup UFSCar (São Carlos). 2014;22(3):5617. doi: $10.4322 /$ cto. 2014.078 .

9. Rocha ANDC, Deliberato D, Araújo RCT. Procedimentos para prescrição dos recursos de tecnologia assistiva para alunos da educação infantil com paralisia cerebral. Rev Educ Esp. 2015;28(53):691-208. doi:10.5902/1984686X14398.

10. Sameshima FS, Silva FRP. Implementação de recursos e procedimentos da tecnologia assistiva no atendimento educacional especializado. Rev Cient Unisalesiano. 2012;3(6). Disponível em: http://www.salesianolins.br/universitaria/ artigos/no6/artigo 13.pdf

11. Scherer MJ, Sax C, Vanbiervliet A, Cushman LA, Scherer JV. Predictors of assistive technology use: the importance of personal and psychosocial factors. Disabil Rehabil Assist Technol. 2005;27(21):1321-31. doi: 10.1080/09638280500164800.

12. Gil AC. Estudo de caso: fundamentação científica, subsídios para a coleta e análise de dados, como redigir o relatório. São Paulo (SP): Atlas; 2009.

13. Bardin L. Análise de conteúdo. Lisboa, Portugal: Edições 70; 2009.

14. Manzini MG, Assis CP, Martinez CMS. Contribuições da Terapia Ocupacional na área da comunicação suplementar e/ ou alternativa: análise de periódicos da Terapia Ocupacional.
Cad Ter Ocup UFSCar (São Carlos). 2013;21(1):59-73. doi: 10.4322/cto.2013.010.

15. Costa CR, Ferreira FMRM, Bortolus MV, Carvalho MGR. Dispositivos de tecnologia assistiva: fatores relacionados ao abandono. Cad Ter Ocup UFSCar (São Carlos). 2015;23(3):61124. doi: https://doi.org/10.4322/0104-4931.ctoAR0544.

16. Rocha PFA, Bohels AE, Silva AMF. Rotina de cuidados de famílias de crianças com paralisia cerebral. Rev Enferm UFSM. 2015;5(4):650-60. doi: http://dx.doi.org/10.1590/01044060.44688 .

17. Bittencourt ZLC, Cheraid DC, MontilhaRCI, Gasparetto MERF. Expectativas quanto ao uso de tecnologia assistiva. J Res Spec Educ Need. 2016;16(1):492-6. doi: 10.1111/14713802.12311 .

18. Ferreira RS, Sampaio PYS, Sampaio RAC, Gutierrez GL, Almeida MAB. Tecnologia assistiva e suas relações com a qualidade de vida de pessoas com deficiência. Rev Ter Ocup Univ São Paulo. 2017;28(1):54-62. doi: 10.11606/issn.22386149.v28i1p54-62.

19. Silva MR, Airoldi MJ. A influência do familiar na aquisição de habilidades funcionais da criança com deficiência visual. Rev Ter Ocup Univ São Paulo. 2014;25(1):36-42. doi: 10.11606/ issn.2238-6149.v25i1p36-42.

20. Bambara JK, et al. Family functioning and low vision: a systematic review. J Vis Impair Blind, Birmingham. 2009;103(3):137-49. Available from: https://www.ncbi.nlm. nih.gov/pmc/articles/PMC2798155/pdf/nihms159048.pdf.

21. Alves ACJ. A tecnologia assistiva como recurso à inclusão escolar de crianças com paralisia cerebral [dissertação]. São Carlos: Universidade Federal de São Carlos; 2009. Disponível em: https://repositorio.ufscar.br/handle/ ufscar/3010?show=full.

22. Johnson JM, Inglebret E, Jones C, Ray J. Perspectives of speech language pathologists regarding success versus abandonment. Augment Altern Commun. 2006;22(2):85-99. doi: 10.1080/07434610500483588.

23. Pape TLB, Kim J, Weiner B. The shaping of individual meanings assigned to assistive technology: a review of personal factors. Disabil Rehabil Assist Technol. 2002;24(1/2/3):5-20.

24. Verza R, Carvalho ML, Battaglia MA, Uccelli M. An interdisciplinary approach to evaluating the need for assistive technology reduces equipment abandonment. J Mult Scler (Foster City). 2006;12(1):88-93. Available from: http://journals. sagepub.com/doi/abs/10.1191/1352458506ms1233oa.

25. Hohmann P, Cassapian MR. Adaptações de baixo custo: uma revisão de literatura da utilização por terapeutas ocupacionais brasileiros. Rev Ter Ocup Univ São Paulo. 2011;22(1):108. Disponível em: http://www.revistas.usp.br/rto/article/ view/14115. 